\title{
Arquitectura de indicadores estratégicos para elevar la calidad de los servicios médicos de los hospitales de alta especialidad
}

\author{
Architecture of strategic indicators to improve the quality \\ of medical services of high specialty hospitals
}

\begin{abstract}
Martha Katty Torres Salgado*凶, Carlos Arturo Vega Lebrún**
Torres Salgado, M. K., \& Vega Lebrún, C. A. (2018). Arquitectura de indicadores estratégicos para elevar la calidad de los servicios médicos de los hospitales de alta especialidad. Investigación y Ciencia de la Universidad Autónoma de Aguascalientes, 26(73), 84-94.
\end{abstract}

\section{RESUMEN}

El presente trabajo tiene como objetivo proponer la construcción de un modelo de indicadores prioritarios de gestión de procesos médicos a través del análisis de las actividades para mejorar la atención médica de calidad y seguridad al paciente en hospitales de alta especialidad (HAE). Estudio de enfoque cuantitativo en una investigación estructurada de manera secuencial y con razonamiento deductivo (Hernández de Sampieri, Fernández Collado, \& Baptista Lucio, 2015). La conclusión de esta investigación afirma que el modelo de indicadores prioritarios de gestión de procesos médicos a través del análisis de las actividades mejora la atención médica de calidad y seguridad al paciente en (HAE), resultado de las premisas investigadas.

El valor agregado es generalizar los datos de una muestra a una población representativa del servicio cirugía y perioperatorio en hospitales

Palabras clave: modelo de indicadores; arquitectura de procesos; servicio de medicina perioperatoria; hospitales de alta especialidad; medidor de desempeño (KPI).

Keywords: indicator model; process architecture; perioperative medicine service; high specialty hospitals; key performance indicator (KPI).

Recibido: 3 de abril de 2017, aceptado: 13 de noviembre de 2017

* Centro de Investigaciones Interdisciplinarias en Ciencias y Humanidades, UNAM. Cto bicipuma 3000, C. P. 04360, C. U., Ciudad de México, México. Correo electrónico: katty_torres_salgado@hotmail. com ** Calidad de Posgrado, Universidad Popular Autónoma del Estado de
Puebla. 21 Sur 1103, C. P. 72410, Barrio Santiago, Puebla, México. Correo electrónico: carlosarturo.vega@upaep.mx

$凶$

Autor para correspondencia privados de alta especialidad de la Ciudad de México, muestra por conveniencia inducida, en tiempo y por accesibilidad a tres nosocomios. Es una investigación de alcance correlacional para evaluar el grado de asociación entre variables, para establecery definir los medidores de desempeño (KPI, en inglés) variables que puedan relacionar su nivel de prioridad y comportamiento en la construcción propuesta en razón de mayor importancia de las actividades (críticas) en los distintos servicios de atención médica.

\section{ABSTRACT}

The objective of this work is to propose the construction of a model of priority indicators for the management of medical processes through the analysis of activities to improve quality medical care and patient safety in high specialty hospitals (HAE, in Spanish). The study adopts a quantitative approach in a structured research to sequential manner, with deductive reasoning (Hernández de Sampieri et al., 2015). The conclusion of this research affirms that the model of priority indicators of medical process management through the analysis of activities improves quality medical care and patient safety in (HAE), as a result of the premises investigated.

The added value is to generalize the data from a sample population, representative of the surgery and perioperative service in high specialty private hospitals of Mexico City, a sample chosen on the basis of convenience of time and accesibility to three hospitals. The study can be used to make a correlation that will evaluate the degree of association between variables, to establish and define the (KPI) variables that can relate their level of priority and behavior 
in the proposed construction due to the greater importance of the (critical) activities in the different services of medical attention.

\section{INTRODUCCIÓN}

La Organización Mundial de la Salud establece que el sistema sanitario está constituido por todas las organizaciones, instituciones, personas y acciones cuya primera finalidad es promover, mantener 0 restaurar la salud cuyo resultado se ve reflejado en la productividad y calidad de vida de un país (Meena \& Thakkar, 2014). En las últimas décadas se promueve atención de alta calidad y seguridad al paciente centrada en el usuario o cliente cuando se realizan diferentes actividades encaminadas a garantizar los servicios de salud (Berenguer Govarnaluses, Mediaceja Álvarez, \& Montoya Medina, 2014).

Un número considerable de organizaciones manifiestan dificultades con la estrategia de ejecución, la falta de estructura y la transparencia en la gestión estratégica para lograr una descripción estructurada de la organización (Simon, Fischbach, \& Schoder, 2014). Los mayores éxitos de los estudios de casos en la gestión de rendimiento hablan de medidas que giran en torno a un aumento de la capacidad para gestionar la estrategia, comprender la organización o evaluar el desempeño (Grey, Perkins, \& Remmers, 2014). La innovación en la gestión hospitalaria es una necesidad actual para garantizar que los procesos de mejora continua tengan un impacto en la seguridad, calidad y atención al paciente. Los programas de excelencia operacional e implementación en hospitales han mostrado ser útiles para gestionar un cambio de cultura orientado a la estandarización, la disminución de desperdicio y el reconocimiento de las aportaciones de los empleados de la institución (Velázquez Hernández \& Yacaman Handal, 2014).

Afirma Artaza Barrios (2011) que en la práctica los modelos de atención no están centrados en los usuarios, se enfocan en la recuperación de la enfermedad y mayor consumo de tecnología. Se observa el interés de que se realicen trabajos de investigación, menciona Rojas González (2014) que se ha realizado una revisión de publicaciones del tema, cuyos resultados justifican la necesidad de implantar modelos de gestión por procesos que normalicen las actuaciones y disminuyan la variabilidad con el fin de brindar a los pacientes la mayor calidad y seguridad.
Artaza Barrios (2011) menciona la necesidad de conducir procesos de cambio, cuyo enfoque radique en generar condiciones institucionales para integrar los procesos asistenciales como una cadena de pasos interdependientes y cooperativos capaces de sostener eficientemente procesos asistenciales de calidad y seguridad al paciente, alineados con las políticas de carácter nacional. Vinot (2014) comenta que la gestión clínica en los hospitales implica una reestructuración de profesionalidad tradicional, mediante la coordinación, la cooperación del trabajo conjunto. En México, el Centro Médico ABC a través de Díaz (2014) señala que los hospitales funcionan de manera fraccionada, cada departamento constituye un silo autónomo que opera de manera desintegrada con seguimiento exhaustivo de indicadores financieros, operativos o clínicos.

A pesar de esfuerzos dirigidos presentados por los autores se han hecho propuestas de forma aislada para algunos servicios médicos y no de forma integral; Meena \& Thakkar (2014) aplicaron cuadro de indicadores balanceados (BSC) con el enfoque integrado de las técnicas de modelado estructural para identificar los indicadores de desempeño y sus relaciones en un sistema de salud de la India. Berenguer Govarnaluses et al. (2014) realizaron estudio descriptivo y transversal durante 2013 para evaluar el grado de satisfacción de cinco proveedores de salud bucal pertenecientes al Policlínico Universitario "Josué País García" de Santiago de Cuba, mediante criterios, indicadores y estándares. Díaz (2014) implementó la Línea de Servicio de Neurología y el impacto en indicadores de gestión en el Centro Médico ABC, para demostrar que un hospital con una estructura de líneas de servicio y médicos organizados tipo «práctica médica grupal» registra un mejor desempeño operativo y clínico que los estructurados por departamentos y práctica médica individual.

Groene, Kringos y Sunol (2014) contribuyen con una metodología propia, para guiar en los hospitales y apoyar el análisis de los procesos de medición cuantitativa. Las siete maneras de mejorar la calidad de seguridad son:

1. Alinear los procesos de organización con la presión o entidades externas.

2. Poner la calidad en la agenda (planes y programas de trabajo). 
3. Implementar sistemas de toda la organización de apoyo para mejorar la calidad.

4. Asegurar responsabilidades y experiencia por equipos a nivel departamental.

5. Organizar las vías de atención basada en la evidencia de intervenciones de calidad y seguridad.

6. Implementar sistemas de información.

7. Realizar una evaluación regular y proporcionar información.

Es importante citar el análisis en recientes cambios en la administración de los hospitales públicos de Francia, y en el mundo, según la reforma promulgada en 2009, dirigida a reforzar el rol administrativo de los doctores. A menudo se argumenta que el liderazgo clínico de alta calidad es un factor clave para el éxito de la organización (Vinot, 2014). Para concluir en este apartado, se puede observar que se han hecho propuestas interesantes y si hay soluciones de forma aislada a través de intentos de medición pero no de forma integral. La aplicación el BSC es lo más utilizado y, por otro lado, liderazgo y herramientas de calidad para mejora de resultados en estos estudios. Meena y Thakkar, que también hacen aplicación de BSC con la innovación de 14 indicadores y mapa de estrategia en el análisis de los procesos hospitalarios, no se analizan en su detalle, solamente como parte de las perspectivas que incluye esta aplicación.

A pesar de esfuerzos bien dirigidos presentados por los autores, se han hecho propuestas interesantes de forma aislada para algunos servicios médicos y no de forma integral en el proceso; por lo cual existe el problema de una carencia de análisis en los procesos de las actividades a detalle, garantizando los resultados $y$, por ende, la priorización de los indicadores en la arquitectura integral de los procesos (AP) de los servicios médicos y de apoyo, haciendo extensivo no sólo a servicios médicos sino mediante la integración y alineación de la estructura orgánica para establecer sus indicadores de forma paulatina, de importancia por niveles de mando y de operación en los hospitales de alta especialidad (HAE).

\section{MATERIALES Y MÉTODOS}

Este trabajo tiene como objetivo proponer un modelo de indicadores prioritarios de gestión de procesos médicos a través del análisis de las actividades para mejorar la atención médica de calidad y seguridad al paciente en HAE. Es una investigación de alcance correlacional, para evaluar el grado de asociación entre variables, establecer y definir las variables (KPI) que puedan relacionar su nivel de prioridad y comportamiento en la construcción propuesta en razón de mayor importancia de las actividades críticas en los distintos servicios de atención médica.

En cada hospital visitado se hizo una sensibilización e involucramiento, para lograr cierto compromiso y responsabilidad social de participación de los cuerpos directivos de hospitales y expertos académicos en especialidades médicas de la Universidad Autónoma Metropolitana (UAM).

\section{Los objetivos específicos fueron:}

- Identificar las actividades prioritarias o concretas en el mapeo de procesos, mediante el prediagnóstico de entidades externas al proceso y alineamiento estratégico para cumplir sus objetivos en el marco de la gestión del sector salud.

- Determinar los mecanismos de articulación de actividades externas que inciden en la satisfacción de los clientes y/o usuarios internos y externos en beneficio del paciente y población demandante del servicio médico de calidad y seguridad al paciente.

- Identificar KPI para el diseño de modelo de indicadores categorizados de calidad y seguridad al paciente.

- Ponderar y priorizar indicadores estratégicos de la alta dirección y de desempeño de acuerdo con los procesos y actividades críticas para evaluación de resultados esperados.

- Desarrollar mejores prácticas de los procesos médicos con calidad y seguridad al paciente, a través del establecimiento de indicadores de desempeño en el servicio de calidad.

- Validar mejores prácticas de los procesos médicos con calidad y seguridad al paciente.

\section{La hipótesis $(\mathrm{H} 1)$ se refiere a:}

Los indicadores en actividades prioritarias o concretas de los procesos médicos y clínicos tienen correlación con la calidad y seguridad ofrecida al paciente en los servicios en la atención hospitalaria de alta especialidad.

\section{Diseño del instrumento de medición}

En la AP se utilizaron y se hicieron diagramas interdisciplinarios para establecer la secuencia de 
Tabla 1

Definición conceptual y operacional de variables

\begin{tabular}{|c|c|c|c|c|c|}
\hline Objetivo & Variables cuantitativas & $\begin{array}{c}\text { Definición } \\
\text { conceptual y } \\
\text { teórica }\end{array}$ & $\begin{array}{l}\text { Operatividad } \\
\text { de variables }\end{array}$ & Instrumento & $\begin{array}{l}\text { de } \\
\text { ción }\end{array}$ \\
\hline $\begin{array}{l}\text { Diseñar modelo de } \\
\text { calidad y seguridad al } \\
\text { paciente de indicado- } \\
\text { res de gestión de pro- } \\
\text { cesos médicos y de } \\
\text { apoyo médico a tra- } \\
\text { vés del análisis de las } \\
\text { actividades prioritarias } \\
\text { en la atención médi- } \\
\text { ca y de apoyo para el } \\
\text { establecimiento de in- }\end{array}$ & $\begin{array}{l}\text { Variables Independientes: } \\
\text { Indicadores en actividades } \\
\text { prioritarias en modelo por } \\
\text { servicio en convergencia o } \\
\text { interdependencia con KPI, } \\
\text { que afectan en su conjunto } \\
\text { a las Variables Dependientes } \\
\text { o resultados en la Calidad y } \\
\text { seguridad al paciente en los } \\
\text { servicios y atención hospita- } \\
\text { laria de alta especialidad. }\end{array}$ & $\begin{array}{l}\text { Desarrollar un } \\
\text { marco de me- } \\
\text { dición propio } \\
\text { (Groene et al., } \\
\text { 2014) en el ren- } \\
\text { dimiento para } \\
\text { el sistema de } \\
\text { atención de la } \\
\text { salud (Meena \& } \\
\text { Thakkar, 2014). }\end{array}$ & $\begin{array}{l}\text { Nivel de aso- } \\
\text { ciación o co- } \\
\text { rrelación e } \\
\text { influencia de } \\
\text { indicadores } \\
\text { en la calidad } \\
\text { de los servi- } \\
\text { cios y aten- } \\
\text { ción hospita- } \\
\text { laria de alta } \\
\text { especialidad. }\end{array}$ & $\begin{array}{l}\text { Análisis de } \\
\text { arquitectura } \\
\text { en mapeos, } \\
\text { encuestas y } \\
\text { entrevistas a } \\
\text { los actores y } \\
\text { reportes en } \\
\text { los servicios } \\
\text { hospitalarios } \\
\text { de evalua- } \\
\text { ción en sito. }\end{array}$ & $\begin{array}{l}\text { Servicios médi- } \\
\text { cos y de apo- } \\
\text { yo en HAE. } \\
\text { Servicio de me- } \\
\text { dicina periope- } \\
\text { ratoria, cirugía } \\
\text { o atención qui- } \\
\text { rúrgica (QX), } \\
\text { enfermería qui- } \\
\text { rúrgica (QX) } \\
\text { y urgencias. }\end{array}$ \\
\hline
\end{tabular}
peño en el servicio de calidad y seguridad al paciente en hospitales de alta especialidad.

Nota: Elaboración propia.

actividades, con el objetivo de que oriente a la comprensión del proceso e identificación de las actividades. Para ello se describe el movimiento o flujo (que se efectúa en un tiempo continuo) a través de esquemas hechos en una hoja de papel o página electrónica de pantalla (Information, 2013). Se optó por la opción de Visita en Sitio, que consistió en ir a las áreas de trabajo y entrevistar a los diversos actores desde el inicio hasta concluir proceso.

Se tienen variables independientes (supuesto o causa a manipular) Entradas. Son los indicadores establecidos en su conjunto, 18 indicadores médicos (Gironés Muriel et al., 2013) para el proceso de cirugía y perioperatorio, que afectan los resultados de calidad en el servicio médico que fueron utilizados para apoyar el análisis con procesos de medición cuantitativa. Y variables dependientes (observado) Salida o resultado (influye y/o mejora). La calidad y seguridad al paciente y seguridad en el servicio y atención hospitalaria de alta especialidad (tabla 1).

\section{Diseño Modelo de indicadores y arquitectura de procesos (AP) en hospitales de alta especialidad} (HAE)

Los modelos son, al fin y al cabo, hipótesis de trabajo, un intento de interpretar y describir de forma esquemática la realidad (Heredia Álvaro, 2001). El modelo explicado a continuación se desarrolla con la AP bajo el concepto de una secuencia de actividades para ofrecer la atención médica en diferentes productos o servicios médicos con valor único al paciente o usuario, a través de la AP y con el uso de mapas e indicadores categóricos que permite integrar paulatinamente a las diversas áreas médicas o de apoyo de la estructura organizacional.

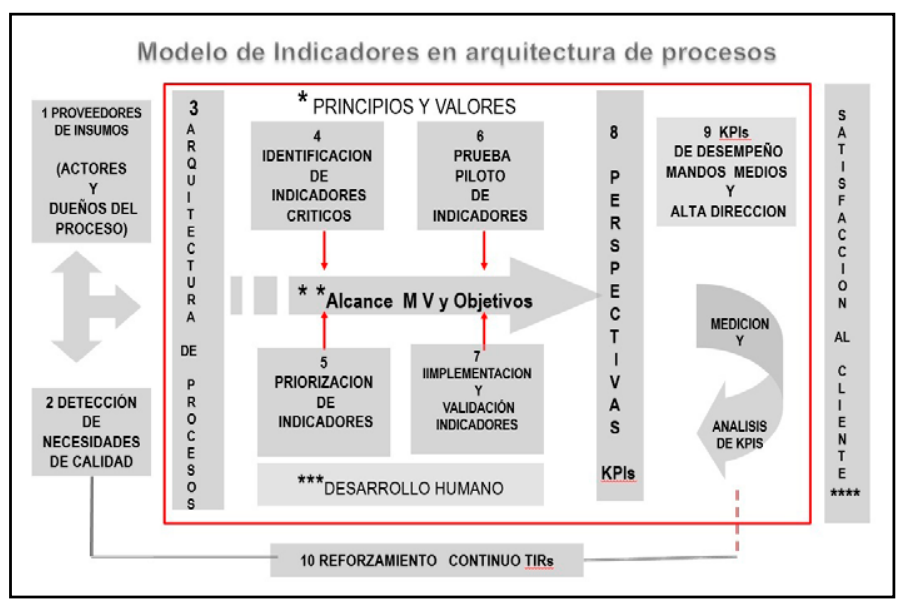

Figura 1. Modelo de indicadores y arquitectura de procesos en hospitales de alta especialidad.

Elaboración propia.

1.Proveedores de insumos (actores y dueños del proceso). Se sigue dependiendo en gran medida de las universidades y escuelas o institutos de nivel medio y superior, lo que se produce en los procesos de enseñanza, que ingresa principalmente en los hospitales, clínicas, sanatorios y consultorios; médicos generales, especialistas y personal de apoyo médico. 
IIVESTIGACIÓn Y CIERCIA DE LA UNIVERSIDAD AUTÓNOMA DE RGUASCALIERTES mapas de procesos interdisciplinarios y transversales con base en entrevistas a los actores del proceso sobre las actividades que realizan.

- Rediseño de actividades. Se rediseña el proceso a través del análisis de cada una de las actividades, se identifican actividades de prioridad y análisis de categorización y de valor al cliente y a la organización (Hammer \& Champy, 1994).

- Modelado de AP. La arquitectura es diseño estructural. AP es el diseño de la estructura de un modelo de procesos que visualiza su configuración de cadena de valor. Este modelo permite vincular estrechamente la disgregación de los subprocesos o etapas del proceso operativo, lo que representa el nivel de estandarización e integración de los procesos de negocio y puede ser considerado como una visualización detallada y general de cómo una organización permitirá y ejecutará estrategias (Simon et al., 2014).

4.Identificación de Indicadores. La construcción de la arquitectura de indicadores (también conocidos por KPl, por sus siglas en inglés) en procesos prioritarios de actividades concretas se basa en los procesos, subprocesos y etapas en la obtención de los mapas de procesos.

La elección de actividades de prioridad concretas. Se elige la actividad de mayor importancia dentro del mismo, no necesariamente en los insumos o resultados del proceso para actividades de prioridad o concretas, lo que permite de manera preventiva garantizar el resultado esperado del bien o servicio.

actividades y su agrupación en niveles; elaborar los

\section{Proceso}

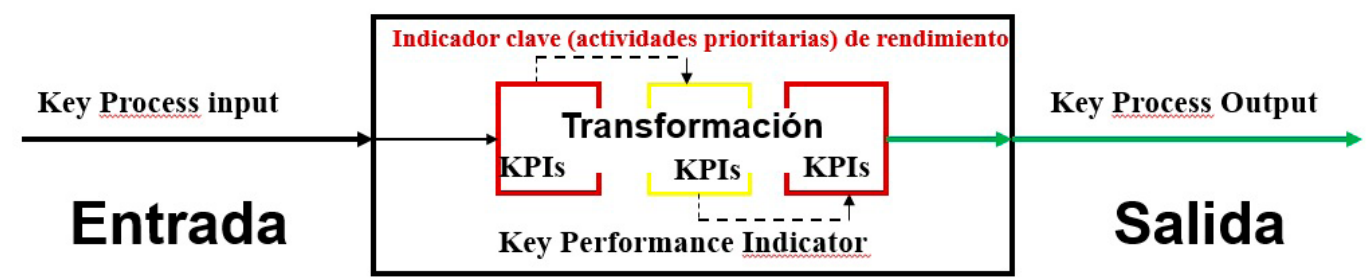

Figura 2. KPI o Indicadores de desempeño para actividades de prioridad o concretas del proceso. (key process input (indicadores de entrada del proceso) y key process output (indicadores de salida o resultados del proceso). Elaboración propia. 
Las actividades de prioridad se identifican claramente en la cadena de valor como actividades únicas y distintivas (Porter, 2011), que entregan de forma satisfactoria el bien o servicio del proceso, o de lo contrario estas actividades se convierten en un cuello de botella que impactaría en la entrega de un bien o servicio insatisfactorio y de conflictos entre las personas. .

Definición de indicadores KPI. El identificar indicadores prioritarios y definir KPI que arrojen mediciones útiles, precisas, correctas e importantes es garantía de llevar una buena estrategia y tomar decisiones correctas y relevantes. Los KPI son indicadores clave en el desempeño y desarrollo de un proceso que permite medir que las actividades se realicen a través de acciones estratégicas. El $\mathrm{KPI}$ ofrece la visualización de las actividades para anticiparse a los resultados y poder tomar las decisiones desde cualquier área funcional en que se ubique la actividad.

5.Priorización de indicadores. Para realizar la categorización de indicadores se utiliza la matriz de priorización (Meena \& Thakkar, 2014). A continuación se detalla: 1. Seleccionar un proceso, diseñar sU AP y especificar su objetivo para analizar sus indicadores de alcance a la entrega del bien o servicio o problema a solucionar. 2. Hacer una lista de los indicadores que estén afectando la calidad a la producción o el servicio (mínimo 15). 3. Definir en forma breve en qué consiste cada uno. 4. Ubicar los indicadores detectados. El número tanto horizontal como vertical corresponderá al mismo problema que permite a través del indicador visualizar para alcanzar el objetivo del proceso. 5. Determinar el nivel de prioridad de una variable sobre otra marcando: "0" si la influencia es Nula "1" si la influencia es Potencial. 6. Hacer la sumatoria tanto por filas como por columnas. 7. El puntaje obtenido de las filas da el Índice de movilidad que indica la prioridad que tiene una variable sobre las demás. Sacar el peso porcentual tomando la sumatoria como 100\%. 8. El puntaje obtenido al sumar las columnas da el Índice de dependencia de una variable con respecto a las otras. 9. Realizar cuadro de porcentajes de movilidad y dependencia para establecer una división y delimitar la zona de prioridad, se divide 100 entre el número de variables y el valor obtenido servirá para trazar una línea vertical y otra horizontal que divida la gráfica para distinguir: zona de prioridad= superior izquierda; zona de análisis de variables o conflicto= superior derecha. Zona de salida del proceso= inferior derecha (key process outputs) y zona de indicadores aislados= inferior izquierda que pueden ser analizados en otro contexto y se hace el análisis.

6.Prueba piloto de indicadores. El propósito del indicador es medir la influencia de variables que afectan los productos o servicios y en su conjunto los resultados de calidad en el servicio o producto que se proporciona. En esta prueba de indicadores se seleccionarán aspectos de importancia y accesibilidad en las áreas o servicios médicos de cirugía, enfermería quirúrgica y en el servicio de medicina perioperatoria (SMPO) y por otro lado directores del área médica, clínica y dirección general de los HAE que tuvieron el interés de documentar controles experimentales e implantación del modelo propuesto y en su caso para hacerlo perfectible y ajustarlo.

7.Implementación de indicadores y validación. Un documento que permite estandarizar los principales elementos que integran el indicador es el diseño de ficha de indicadores tipo, que permite dar seguimiento a través del monitoreo por el actor - dueño responsable de reportar los avances. Además de evidenciar y validar si el control establecido es el idóneo y funciona para recopilar la información estadística para consolidar su ajuste e implementación e incorporarlo como parte del tablero para el grupo operativo o bien para la alta dirección.

8.Perspectivas de indicadores. La categorización se fundamenta en Simon et al. (2014), lo cual permitirá agruparlos de acuerdo con este criterio para visualizar el avance de los objetivos de cada grupo de indicadores de manera puntual.

Rentabilidad (productividad). Se refiere a recuperar la inversión y obtener beneficios financieros en costo/ beneficio. Para los hospitales es común utilizar el término categórico de productividad que se refiere a resultados en los servicios de atención médica.

Actividades. Eslabón prioritario o crítico dentro de la cadena de valor para la obtención de un bien o servicio (Simon et al., 2014).

Seguridad al paciente. Clasificación utilizada para indicadores que monitorean el uso de buenas prácticas preventivas para disminución de fallas, que son causas de eventos adversos, en el otorgamiento de atención al paciente (Meena \& Thakkar, 2014). 
IIVESTIGACIÓn Y CIERCIA DE LA UNIVERSIDAD AUTÓNOMA DE RGUASCALIERTES
90

Oferta. Son las propuestas de valor que se ofrecen al cliente o usuario, en sus productos/servicios.

Calidad. Abarca la satisfacción al cliente o usuario en los bienes o servicios que recibe.

Instancias regulatorias de salud. Indicadores que designan como obligatorias entidades externas sanitarias y de seguimiento de observancia obligatoria en beneficio de la calidad y seguridad ofrecidas al paciente.

9.KPI de desempeño a mandos medios y alta dirección. Los tableros estratégicos KPI son una herramienta diseñada en una estructura matricial. Se diseñaron con los mismos elementos por niveles (SAP, 2004), tanto para alta dirección nivel 1 logro de procesos clave o de prioridad y para mandos medios nivel 2, lo único que cambia es la priorización de los indicadores en su visualización. En la parte lateral de la matriz se establecen las diversas jerarquizaciones de categorización nombradas "Perspectivas" y seleccionadas por los actores de los procesos. En la parte superior derecha se incluyen los datos relevantes del KPI que se pretende visualizar después de ser medidos. La descripción de su diseño estructural:

Perspectiva. Permite categorizar los indicadores: Rentabilidad (productividad), Actividades, Seguridad al paciente, Oferta, Calidad e Instancias regulatorias de salud.

Áreas médicas. Especifica el área de la estructura orgánica del puesto.

Objetivo. Identifica y expresa cualitativamente las finalidades hacia las cuales deben dirigir los recursos,

\footnotetext{
Indicadores de Mandos Medios Qx y SMPO:

18-DCOM Porcentaje en la disminución de complicaciones quirúrgicas: 16+2 Cirugía de más de $2 \mathrm{~h}$ de duración; $13 \mathrm{MOR}$ Mortalidad dentro de Quirófano; 11CAN Porcentaje de pacientes que presentaron complicaciones de la anestesia en relación con el total de intervenciones quirúrgicas; 12REI Porcentaje de intervenciones quirúrgicas dentro de las $72 \mathrm{~h}$ inmediata a la primera intervención en relación con el total de reintervenciones quirúrgicas; $3 \mathrm{CC}$ Cirugías canceladas; 17CUASI Número de errores, cuasifallas y omisiones para garantizar cirugía correcta; 9EP Porcentaje de pacientes que presentaron embolia pulmonar en relación con el total de intervenciones quirúrgicas; 1OSEPS Porcentaje de pacientes que presentaron sepsis postoperatoria en relación con el total de intervenciones quirúrgicas; $2 E$ Eficacia de cirugías en tiempo programado; $8 \mathrm{P} 10$ Productividad quirúrgica (10 Qx más frecuentes), distribución por edad y grupo etareo; 7DQ Porcentaje en diferimiento quirúrgico; $5 \mathrm{CA}$ Cirugías realizadas por aseguradora; 4CE Cirugías por especialidades Qx; 3CC Cirugías canceladas; 15LAP Porcentaje quirúrgico laparoscópicas que terminan abiertas; $14 \mathrm{AB}$ Porcentaje de apendicitis blancas; 6CS Número de intervenciones quirúrgicas por sala.
}

esfuerzos y actividades de la organización para dar cumplimiento a su misión y visión.

Estrategia. Iniciativa que establece cómo alcanzarán el objetivo y la meta que se ambiciona para obtener los resultados esperados.

Indicador KPI. Medio, vínculo o variable aleatoria para medir valores en una escala de medición derivados de serie de hechos observados en las actividades de prioridad de los procesos.

Resultados del indicador. Estos cobran sentido de acuerdo con los objetivos establecidos (resultados esperados) a partir de los cuales se organiza una serie de actividades para alcanzarlos. Incluye: 1. Meta. Es un elemento (s) cuantitativo del objetivo en tiempo. 2. Status. Es la parametrización de acuerdo con el avance de los resultados y se le asigna un color verde, rojo o amarillo dependiendo del límite máximo o mínimo permisible.

10.Reforzamiento Continuo TIR. Son las buenas prácticas entre sus colaboradores y una retroalimentación activa que mantiene motivado al personal para generar en el mismo un alto nivel de compromiso.

\section{RESULTADOS}

Se presenta a continuación el proceso de atención quirúrgica y del servicio de medicina perioperatoria (SMPO), se trabajan de manera simultánea y complementaria; este segundo proceso en periferia a cirugía para garantizar la calidad de atención al paciente desde el preoperatorio hasta su alta de hospitalización en hospitales privados y públicos con grandes retos de innovación médica. El resultado del análisis de priorización es de 18 indicadores' para mandos medios.

Este indicador de complicaciones quirúrgicas y de cobertura en el (SMPO) es dos KPI afectan la calidad y seguridad al paciente. En la primera categoría abarca la satisfacción al cliente o usuario en los bienes o servicios que recibe, y en la segunda se monitorean el uso de buenas prácticas preventivas para disminución de complicaciones quirúrgicas, que son causas de eventos adversos; en el otorgamiento de atención al paciente.

El indicador de Complicaciones Quirúrgicas se implementa para medir y monitorear en esta investigación, para registrar las complicaciones en función de su cobertura de atención y servicio. Previamente identificada la actividad en el mapa 


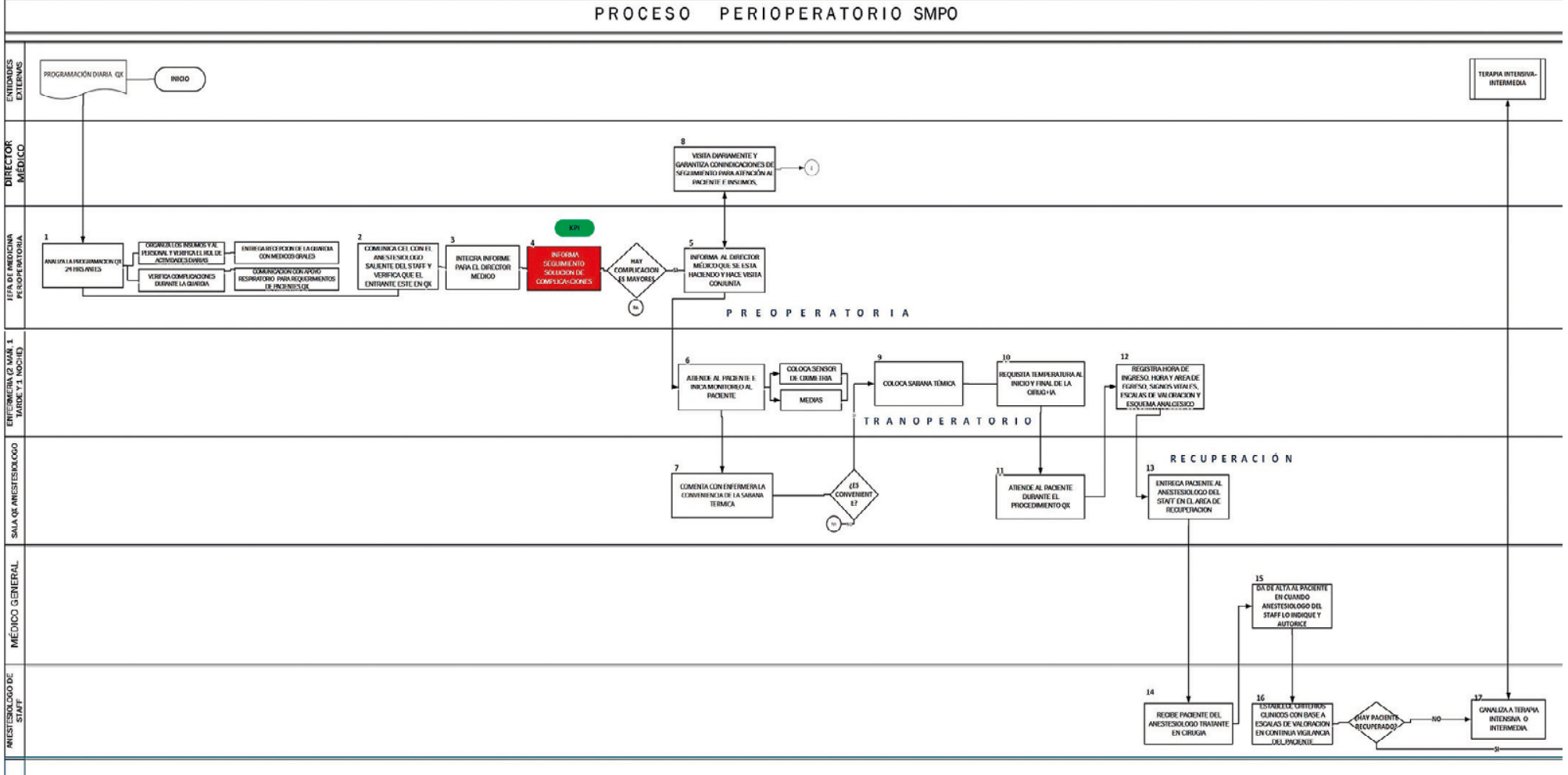

Figura 3. Arquitectura del Proceso (SMPO).

Elaboración propia.

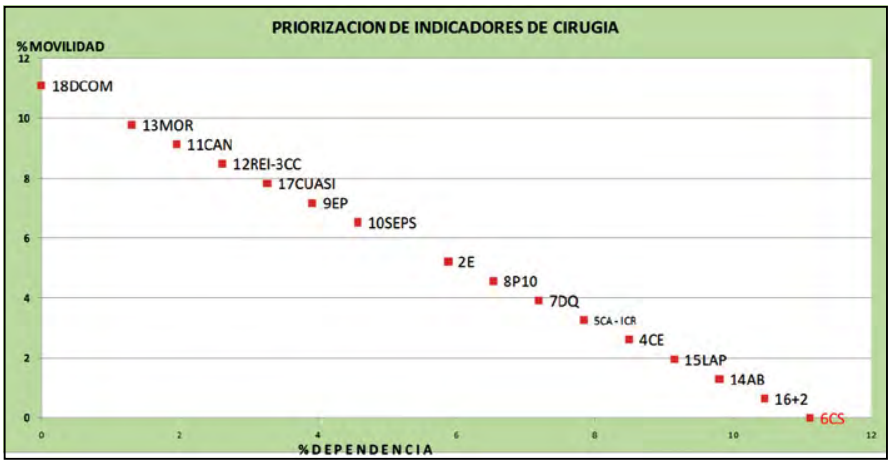

Figura 4. Priorización de Indicadores de QX- SMPO.

Elaboración propia.

del proceso perioperatorio y de atención quirúrgica; el algoritmo es: \% Complicaciones= Número de alteraciones fisiológicas detectadas y resueltas/ Número de pacientes vigilados por el servicio X 100. El $\%$ en complicaciones se obtuvo del análisis mensual del proceso SMPO como se puede ver en la tabla 2 de complicaciones quirúrgicas. El análisis permite contar con información que ayuda a los médicos a encontrar la causa raíz de porque se complica la intervención quirúrgica; por ejemplo si hay muchos pacientes con náuseas posiblemente se tendrá que revisar el medicamento que está causando un
Tabla 2

Complicaciones quirúrgicas (Qxs)

\begin{tabular}{lccccc}
\hline \multicolumn{1}{c}{$\begin{array}{c}\text { Complicaciones } \\
\text { QXs }\end{array}$} & Dic & Ene & Feb & Mar \\
Náuseas & 15 & 16 & 16 & 16 & Total \\
Vómito & 9 & 2 & 5 & 8 & 24 \\
Prurito & 5 & 1 & 1 & 1 & 8 \\
Cefalea & 0 & 0 & 2 & 3 & 5 \\
Bloqueo sensitivo/ & 0 & 0 & 3 & 3 & 6 \\
motor-residual & 0 & 1 & 0 & 0 & 1 \\
Micción & 0 & 1 & 0 & 0 & 1 \\
Fiebre & 0 & 2 & 0 & 0 & 2 \\
Dolor mayor 5 & 7 & 2 & 2 & 5 & 16 \\
Saturación de oxígeno & & & & & \\
menor a 90\% & 0 & 2 & 1 & 1 & 4 \\
Taquicardia & 0 & 0 & 0 & 1 & 1 \\
Bradicardia & 1 & 0 & 1 & 1 & 3 \\
Hipertensión & 0 & 0 & 0 & 1 & 1 \\
Hipotensión & 1 & 0 & 1 & 1 & 3 \\
Sangrado & 1 & 0 & 0 & 0 & 1 \\
Totales & 24 & 11 & 16 & 25 & \\
\hline
\end{tabular}

Nota: Elaboración propia 
PROCESO PERIOPERATORIO SMPO

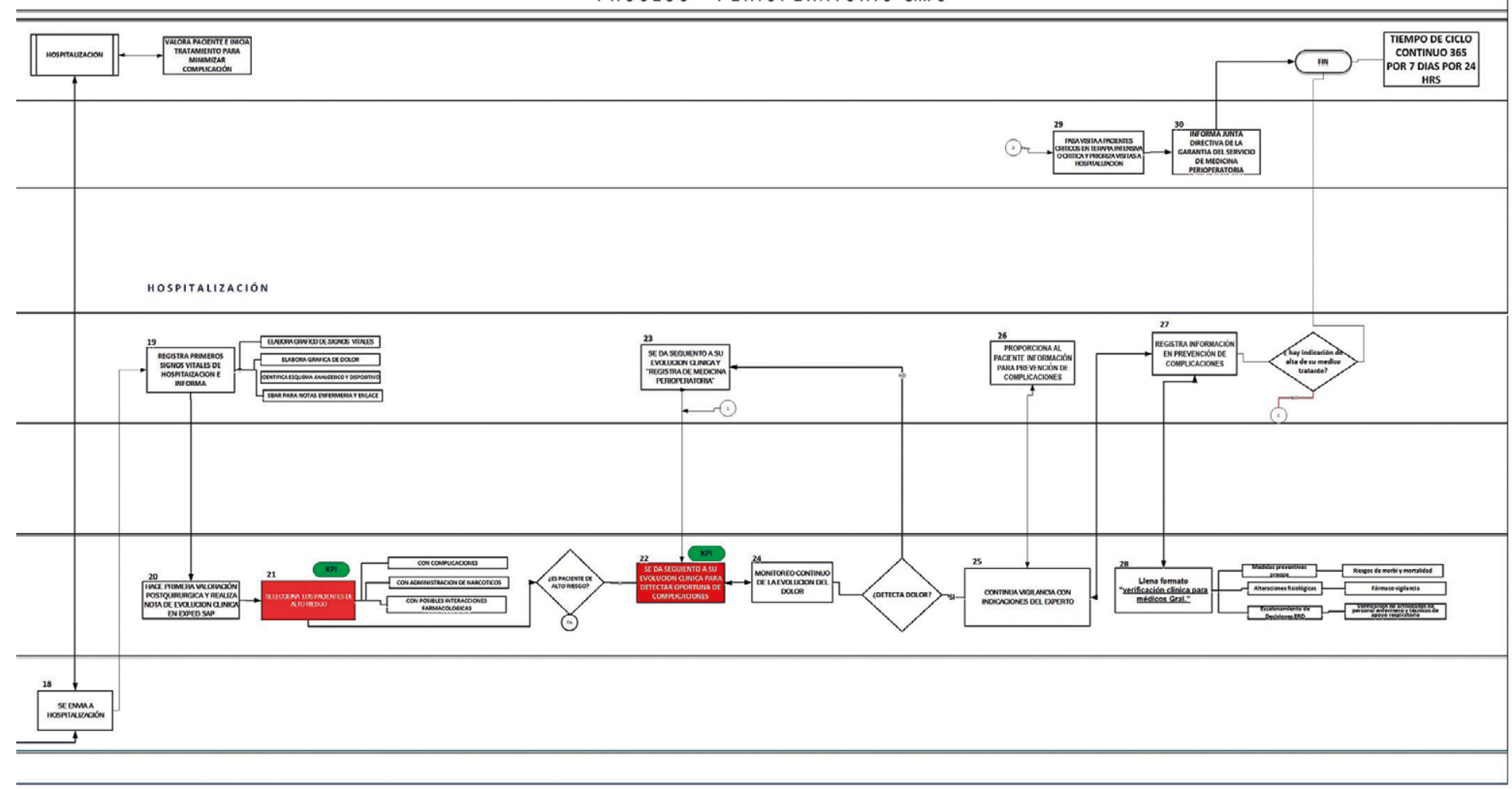

Continuación de la Figura 3. Arquitectura del Proceso (SMPO).

Elaboración propia.

efecto colateral con los pacientes e incrementa los vómitos. Se tendrá que cambiar de inmediato el medicamento. Enseguida se visualizan la cobertura que abarca el proceso de SMPO, se determina su alcance y con ello se valora si están disminuyendo las complicaciones quirúrgicas.

El indicador de la cobertura atendida del (SMPO) se integra como indicador para calcular las complicaciones en función de su cobertura de atención y servicio. Previamente identificada la actividad en el mapa del proceso (SMPO) y de atención quirúrgica, el algoritmos es: \% Cobertura atendida en el SMPO vigilados en el (SMPO)/Cirugías Totales X 100. Dicho indicador muestra cual es la totalidad de pacientes quirúrgicos en el cual se da continuidad en este servicio y refleja la garantía de dar seguimiento a pacientes desde que ingresan a cirugía hasta que son dados de alta. La perspectiva de estos dos indicadores de complicaciones quirúrgicas y de cobertura en el SMPO afecta la calidad y seguridad ofrecida al paciente. El resultado del uso y categorización en el control se muestra en la tabla 3.

\section{DISCUSIÓN}

En la AP se observó como la metodología propuesta permite vincular estrechamente la disgregación de los subprocesos o etapas del proceso operativo, lo que representa el nivel de estandarización e integración de los procesos de negocio y puede ser considerado como una visualización a detalle general de cómo una organización ejecuta estrategias. El objetivo del KPI no es exclusivamente analizar los avances hacia el logro de resultados esperados, sino hacer uso de buenas prácticas que sirvan para el reforzamiento continuo de los diversos actores del proceso y apoyar al equipo de alto desempeño encargado a desarrollar diversas competencias que se verán reflejadas en los resultados que espera la alta dirección.

El modelo incluye desarrollo humano que vincula las prácticas de los recursos, las capacidades de la fuerza de trabajo, los resultados comerciales y desempeño financiero se tendrán que vincular las capacidades del capital humano con resultados empresariales (SAP, 2004) a través de: 
Tabla 3

Porcentaje Cobertura atendida en el SMPO

\begin{tabular}{cccccc}
\hline PERIODO & COMPLICACIONES & $\begin{array}{c}\% \\
\text { COMPLICACIONES } \\
\text { QXS }\end{array}$ & $\begin{array}{c}\text { PACIENTES } \\
\text { PERIOPERATORIOS } \\
\text { VIGILADOS }\end{array}$ & CIRUGÍAS & $\begin{array}{c}\text { \% CUBIERTO } \\
\text { SPO }\end{array}$ \\
\hline Dic-15 & 24.00 & 12.06 & 199 & 229 & 87 \\
Ene-16 & 11.00 & 5.95 & 185 & 224 & 83 \\
Feb-16 & 16.00 & 7.66 & 209 & 237 & 88 \\
Mar-16 & 25.00 & 11.47 & 218 & 231 & 94 \\
\hline TOTALES & 76.00 & & 811 & 921 & \\
\hline
\end{tabular}

Nota: Elaboración propia.

Tabla 4

Tablero Estratégico KPI del (SMPO)

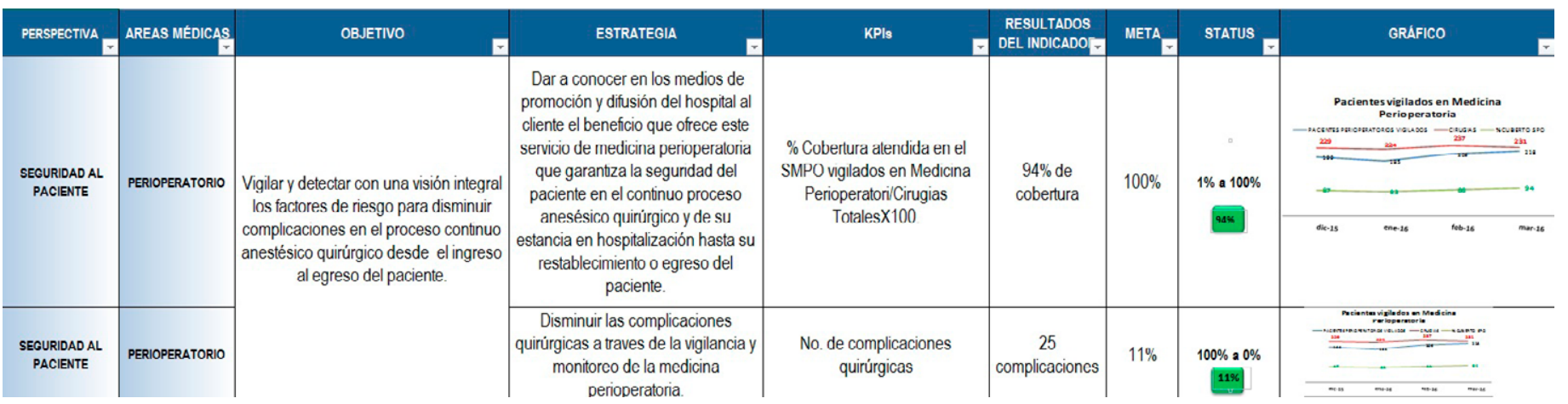

Nota: Elaboración propia.

1. La evaluación diagnóstica que destaca áreas para mejora del rendimiento o creación de valor

2. Medición recurrente de una actividad alineada con los procesos centrales de planificación de una organización.

3. Como parte de una transformación organizativa a gran escala, donde el objetivo es remodelar los recursos humanos tradicionales, el aprendizaje, la formación y las funciones de desarrollo para adaptarlas a una nueva estrategia de negocio.

\section{CONCLUSIONES}

Esta investigación afirma que el modelo de indicadores prioritarios de gestión de procesos médicos a través del análisis de las actividades mejora la atención médica de calidad y seguridad ofrecidas al paciente en HAE, como se observó, aunado a la reducción de costos por este concepto. Se acepta el supuesto de hipótesis a través de un análisis de correlación múltiple de los indicadores en actividades prioritarias de los procesos médicos, clínicos e investigadores especialistas médicosacadémicos de la UAM (Xochimilco) que obtienen correlación de r cuadrado de .950. Por otro lado, es de .309 para mandos medios con relación a la alta dirección; lo cual se interpreta como que los mandos medios tienen la misma prioridad de indicadores que la alta dirección en .30 y el restante obedece a otras prioridades de medición de indicadores de acuerdo a la operatividad cotidiana. El factor de Durbin Watson de 2.215 indica que sí se cumple el supuesto de independencia de errores bajo el criterio de aceptación o rechazo si el valor entra ente 1 y 3; por tanto, se acepta el supuesto de la hipótesis planteada en esta investigación.

Ahora bien, es vital categorizar los indicadores de la alta dirección, lo que es de importancia para ellos como eficiencia y rendimiento. A mandos medios los aspectos médicos de las actividades operativas, que realizan cotidianamente que 
garanticen (prevención) con calidad y seguridad al paciente en los servicios en la atención hospitalaria de alta especialidad. Demuestra que los indicadores deben de categorizarse de acuerdo con el interés de importancia para alta dirección o mandos medios que conducen a la oportuna toma de decisiones basadas en la prevención para garantizar resultados esperados en la calidad de la atención médica.

REFERENCIAS

- Artaza Barrios, O., Holder Morrison, R., Méndez, C., \& Suárez Jiménez, J. (2011). Redes integradas de servicios de salud: El desafio de los hospitales (299 pp.). Santiago, Chile: OPS-OMS.

- Berenguer Gouarnaluses, M. C., Mediaceja Álvarez, N. R., \& Montoya Medina, M. (2014). Evaluación de la satisfacción en salud: Un reto en la atención estomatológica. MEDISAN, 18(7), 946-950.

- Díaz, A. A. (2014). Gestión por líneas de servicio en el Centro Médico ABC. Anales Médicos-Medigraphic, 59(3), 171-178.

- Gironés Muriel, A., Matute, E., González Perrino, C., Serradilla Mateos, L., Molinero, T., \& Mariné, M. (2013). Resultados globales en un hospital privado sobre su modelo. CMA Cirugía Mayor Ambulatoria, 18(2), 61-67.

- Groene, O., Kringos, D., \& Sunol, R. (2014). Seven ways to improve quality and safety in hospitals. An evidence basedguide. Editado por DUQUE. Recuperado de www.duque.eu

- Hammer, M., \& Champy, J. (1994). Reingeniería. Olvide lo que sabe sobre cómo debe funcionar una empresa. ¡Casi todo está errado! (trad. de la $1^{\text {a. }}$ en inglés J. Cárdenas Nannetti). Bogotá: Grupo Editorial Norma.

- Heredia Álvaro, J. A. (2001). Sistema de indicadores para la mejora y el control integrado de la calidad de los procesos. Castelló de la Plata: Universitat Jaume I-Athenea.

- Hernández de Sampieri, R., Fernández Collado, C., \& Baptista Lucio, P. (2015). Metodología de la investigación (6a. ed.). México: McGraw-Hill Education.

- Information, M. (2013). Manual para desarrollo de procedimientos, politicas y documentacion [Manual de curso]. Info-Maps.

- Meena, K., \& Thakkar, J. (2014). Development of Balanced Scorecard for healthcare using Interpretive Structural Modeling and Analytic Network Process. Journal of Advances in Management Research, 11, 232-256. doi: 10.1 108/JAMR-122012-0051

- Perkins, M., Grey, A., \& Remmers, H. (2014). What do we really mean by "Balanced Scorecard"? International Journal of Productivity and Performance Management, 63(2), 148-169. doi: 10.1108/IJPPM-11-2012-0127

- Porter, M. E. (2009). Ser competitivo. España: Ediciones Deusto. (2010). Ventaja competitiva. Creación y sostenibilidad de un rendimiento superior. México: Pirámide.

(2011). ¿Qué es la estrategia? Harvard Business Review, diciembre 2011, 100-117. Recuperado de http://www. ucipfg.com/Repositorio/MAES/MAES-03/Unidad l/ESTRATEGIA\%20MPORTER\%202011.pdf

- Rojas González, A. (2014). Modelos de gestión por procesos integrados en la salud. Ene (versión en línea), 8(3). doi: 10.4321/ S1988-348X2014000300008

- SAP AG (2004). Human Capital Management. A measurement breakthrough on the horizon (19 pp.) [Libro blanco]. Recuperado de https://es.slideshare.net/Jacknickelson/ human-capital-management-3914776

- Simon, D., Fischbach, K., \& Schoder, D. (2014). Enterprise architecture management and its role in corporate strategic management. Information Systems and e-Business Management, 12(1), 5-42.

- Velázquez Hernández, B. M., \& Yacaman Handal, R. (2014) Implementación de un programa de excelencia operacional como una herramienta para la mejora continua en el Centro Médico ABC. Anales Médicos-Medigraphic, 59(3), 179-184.

- Vinot, D. (2014). Transforming hospital management à la francaise: The new role of clinical managers in French public hospitals. International Journal of Public Sector Management, 27(5), 406-416. doi: 10.1108/IJPSM-06-2012-0067 